\title{
Intergenerational Preference Differences and Optimal National Saving*
}

\author{
JOHN P. LAITNER \\ Department of Economics, The University of Michigan, Ann Arbor, Michigan 48109
}

Received June 19, 1978; revised January 25, 1979

\begin{abstract}
This paper investigates the problem facing national planners who realize that their successors may employ different social welfare criteria for determining economic policies than they do. In such a situation, we show that the present planners may want to leave future generations a smaller capital stock than they would if they thought the stock would be managed in a way consistent with their own preferences. On the other hand, we also identify cases in which the opposite is true. We obtain the results by studying an optimal aggregate growth model the utility function of which changes randomly from one generation to the next. We analyze the model using a system of functional equations in place of the conventional Bellman equation from dynamic programming theory.
\end{abstract}

Optimal growth models (see, for example, [3, 12]) posit the existence of a societal utility function registering a nation's tastes over possible time paths of average per capita consumption. At a given time such a function presumably represents an amalgamation of the preference orderings of existing citizens, the citizens being able to weigh the benefits of alternative levels of present consumption against those of corresponding amounts of consumption for future cohorts.

Customarily optimal growth models adopt two key postulates: (1) each generation's societal utility function is additively separable between time periods and stationary in the Koopmans' [6, postulate 4] sense, and (2) all cohorts have the same function. These assumptions ensure that as one generation adjusts its aggregate saving to maximize national welfare, it is coincidentally behaving exactly as previous generations would have wanted it to. That fact enables modelers to use Bellman's "principle of optimality" to define the indirect utility function of each generation with a single recursive functional equation, the so-called Bellman equation. The equation often provides useful characterizations of society's best bchavior.

* I am indebted to my colleague Alan Deardorff and to two anonymous referees for many helpful comments on earlier drafts of this paper. 
The purpose of this paper is to drop the second assumption listed in the preceding paragraph, asking instead the following question: If succeeding generations might have different preferences, how should the present one behave. Because a society's membership changes over time, logically national preferences could too. But if they do, one cohort cannot assume future societics will consume and save exactly the amounts it would like. So, the Bellman equation will no longer be valid. We show that if national preferences change randomly over time, however, we can develop a system of two simultaneous functional equations replacing Bellman's single one. Employed together, the new equations can determine equilibrium behavior for all generations. Under restrictive, but familiar, additional hypotheses we exhibit a solution to the system of equations and show that in various cases intergenerational preference variability unambiguously raises or lowers optimal aggegate saving.

\section{THE MODEL}

The problem of how much one cohort is to save if it knows its descendants may have different preferences seems very difficult. In order to make any headway, therefore, we make the following assumptions.

First, we assume each generation's societal (von Neumann-Morgenstern) preference ordering is a stationary member of the Bergson class:

$$
U=\sum_{i=0}^{\infty} h^{i} \cdot u\left(C_{i}, b\right)
$$

with

$$
u(C, b)=b \cdot C^{b}, \quad b \in(-\infty, 0) \cup(0,1){ }^{1}
$$

The variable $C_{0}$ measures average per capita consumption for the present generation, $C_{1}$ is average per capita consumption for the next, and so on. We will often find $\sigma=\sigma(b)=1 /(1-b)$, negative the inverse of the elasticity of marginal utility with respect to current consumption, more convenient to work with than the constant $b$. We require that the subjective discount factor $h$ be between 0 and 1 .

Second, we assume a linear technology: if $Q_{t}$ is average per capita output at time $t$, then

$$
Q_{t+\mathbf{1}}-\lambda \cdot\left(Q_{t}-C_{t}\right)
$$

${ }^{1}$ We omit the logarithmic, or $b=0$, case. The Appendix and the discussion in Section 3 show that $\boldsymbol{b}=0$ is the dividing line for case-by-case analyses. 
(provided $Q_{t}-C_{t} \geqslant 0$ ). In words, $Q_{t}-C_{t}$ is the capital stock bequeathed per capita at time $t$, and the positive constant $\lambda$ is the average productivity of capital divided by 1 plus the rate of population growth.

Suppose, for the moment, that all generations have the same preferenceordering parameters $h$ and $b$. Then if $v(Q, h, b)$ stands for the maximum possible utility subject to line (3) of a generation with average income $Q$,

$$
v(Q, h, b)=\operatorname{Max}_{S \geqslant 0}\{u(Q-S, h)+h \cdot v(\lambda S, h, b)\} .
$$

In fact, if $h \lambda^{b}<1$,

$$
v(Q, h, b)=b \cdot\left(1 /\left(1-(h \Lambda)^{o}\right)\right)^{1 / \sigma} \cdot Q^{b},
$$

where $A=\Lambda(b)=\lambda^{b}$ (see Levhari and Srinivasan [7]). If $s(Q, h, b)$ gives the unique maximizing value of $S$ for the right-hand side of line (4),

$$
s(Q, h, b)=(h \Lambda)^{\sigma} \cdot Q .
$$

We will use lines (5) and (6) for comparisons in Section 3. To prevent confusion, from this point forward we will name all alternative indirect utility functions and optimal saving rules with capital letters.

\section{Differing Preference Orderings}

We now let every cohort have its own values for $h$ and $b$. For the sake of simplicity we assume that each generation's pair $(h, b)$ is an independent realization from a fixed distribution (although we could develop functional equations for any case in which pairs are generated by a Markov process). We also assume that each cohort makes its consumption-saving decision without knowing the parameter pairs which will apply to future generations, although each cohort does know the distribution from which all pairs are sampled.

The assumption that the sampling distribution for pairs $(h, b)$ does not change over time gives the new model a recursive structure, which we exploit as follows. Let $\left(h_{t}, b_{t}\right)$ characterize the preferences of generation $t$. Let $h_{t} \cdot R\left(\lambda S_{t}, h_{t}, b_{t}\right)$ stand for the discounted expected utility accruing to generation $t$ (vicariously) from the consumption of future cohorts (i.e., from $\left.C_{t+1}, C_{t+2}, \ldots\right)$. Note that the values of $C_{t+1}, C_{t+2}, \ldots$ will depend not only on the average bequest per capita generation $t$ leaves, $S_{t}$, but also on $\left(h_{t+1}, b_{t+1}\right),\left(h_{t+2}, b_{t+2}\right), \ldots$ Because of the stationarity of generation $t$ 's preference ordering, however, its vicarious expected utility from $C_{t+2}, C_{t+3}, \ldots$ must be $h_{t}{ }^{2} \cdot R\left(\lambda \cdot\left(Q_{t+1}-C_{t+1}\right), h_{t}, b_{t}\right)$. The function $R(\cdot)$ needs no time 
subscript because the time horizon is infinite. So, if $S\left(Q_{t+1}, h_{t+1}, b_{t+1}\right)$ gives the saving of generation $t+1$ when its taste parameters are $h_{t+1}$ and $b_{t+1}$, the function $R(\cdot)$ must obey

$$
\begin{aligned}
R\left(Q_{t+1}, h_{t}, b_{t}\right)= & E\left[u\left(Q_{t+1}-S\left(Q_{t+1}, \tilde{h}_{t+1}, \tilde{b}_{t+1}\right), b_{t}\right)\right] \\
& +h_{t} \cdot E\left[R\left(\lambda S\left(Q_{t+1}, \tilde{h}_{t+1}, \tilde{b}_{t+1}\right), h_{t}, b_{t}\right)\right]
\end{aligned}
$$

where $\tilde{h}_{t+1}$ and $\tilde{b}_{t+1}$ are random variables. Note that the present cohort uses $h_{t}$ and $b_{t}$ in all of its utility calculations, although it cannot force subsequent generations to determine their saving rates using the same constants. ${ }^{2}$

Generation $t+1$ determines the function $S(\cdot)$ from its own utility maximization problem. Assuming it uses $h_{t+1} \cdot R\left(\lambda S_{t+1}, h_{t+1}, b_{t+1}\right)$ to compute its vicarious expected utility from $C_{t+2}, C_{t+3}, \ldots$, we have

$$
\begin{aligned}
u\left(Q_{t+1}\right. & \left.-S\left(Q_{t+1}, h_{t+1}, b_{t+1}\right), b_{t+1}\right) \\
& +h_{t+1} \cdot R\left(\lambda S\left(Q_{t+1}, h_{t+1}, b_{t+1}\right), h_{t+1}, b_{t+1}\right) \\
= & \operatorname{Max}_{S \geqslant 0}\left\{u\left(Q_{t+1}-S, b_{t+1}\right)+h_{t+1} \cdot R\left(\lambda S, h_{t+1}, b_{t+1}\right)\right\}
\end{aligned}
$$

Combining (7) and (8), and streamlining the notation slightly, we have a recursive system of two functional equations,

$$
\begin{gathered}
R(Q, h, b)=E[u(Q-S(Q, \tilde{h}, \tilde{b}), b)]+h \cdot E[R(\lambda S(Q, \tilde{h}, \tilde{b}), h, b)], \\
u(Q-S(Q, h, b), b)+h \cdot R(\lambda S(Q, h, b), h, b) \\
=\operatorname{Max}_{S \geqslant 0}\{u(Q-S, b)+h \cdot R(\lambda S, h, b)\},
\end{gathered}
$$

in two "variables," $R(\cdot)$ and $S(\cdot)$.

If $h$ and $b$ are the same for every cohort, the right-hand side of Eq. (9) equals the left-hand side of (10). In that case substitution will reduce the system to the single functional equation of Section 1, Eq. (4). If $h$ and $b$ can vary over time, however, the right-hand side of (9) and the left-hand side of (10) are not necessarily equal, so collapsing the system to one equation is not feasible.

Suppose $(R(\cdot), S(\cdot))$ solves Eqs. (9) and (10). Then as shown below, we can think of $S(Q, h, b)$ as giving the desired saving for a generation with

\footnotetext{
${ }^{2}$ The present generation uses $h_{t}$ and $b_{t}$ in its calculations because its direct utility function (see line (1)) depends on $C_{t}, C_{t+1}, \ldots$ If $U$ depended on $C_{t}$ and the utility of future generations instead, then the present cohort's calculations in line (7) would depend on $\left(\tilde{h}_{t+1}, \tilde{b}_{t+1}\right)$ and $\left(\tilde{h}_{t+2}, \tilde{b}_{t+2}\right)$, but not $\left(h_{t}, b_{t}\right)$.
} 
taste parameters $h$ and $b$ and per capita national product $Q$. The indirect utility function for such a generation is

$$
\begin{aligned}
V(Q, h, b)= & u(Q-S(Q, h, b), b) \\
& +h \cdot R(\lambda \cdot S(Q, h, b), h, b) .
\end{aligned}
$$

To rule out anomalies, however, such as $R(Q, h, b)=\infty$ all $Q$, which satisfies lines (9) and (10) if, for example, $S(Q, h, b)=Q / 2$, we require that to constitute a solution for our model, $(R(\cdot), S(\cdot))$ must satisfy the following restriction in addition to the two functional equations above: for any $Q>0$,

$$
R(Q, h, b)=\sum_{i=1}^{\infty} h^{i} \cdot E\left[u\left(\tilde{C}_{i}, b\right)\right],
$$

where $\tilde{Q}_{0}=Q, \quad \tilde{h}_{0}=h, \quad \tilde{b}_{0}=b$, and $\tilde{Q}_{i}=\lambda \cdot S\left(\tilde{Q}_{i-1}, \tilde{h}_{i-1}, \tilde{b}_{i-1}\right)$ and $\tilde{C}_{i}=\tilde{Q}_{i}-S\left(\tilde{Q}_{i}, \tilde{h}_{i}, \tilde{b}_{i}\right)$ all $i \geqslant 1$. This new restriction comes from line (1) and the definition of $R(\cdot)$.

We could easily modify our functional equations to encompass nonlinear technologies and direct utility functions outside of the Bergson class. The advantage of making the strong assumptions of lines (1)-(3), however, is that, given them, we can often prove the existence of a solution $(R(\cdot), S(\cdot))$ to lines (9), (10), and (12), and, as Section 3 shows, we can compare the resulting saving function $S(\cdot)$ with $s(\cdot)$ from section $1 .^{3}$

To solve the simultaneous equations of lines (9) and (10) let us try a functional form for $R(\cdot)$ similar to the one used in Section 1:

$$
R(Q, h, b)=b \cdot r(h, b) \cdot Q^{b} .
$$

Substituting this into Eq. (10), the optimal saving rule is

$$
S(Q, h, b)=\left(\frac{(h \cdot \Lambda \cdot r(h, b))^{\sigma}}{1+(h \cdot \Lambda \cdot r(h, h))^{\sigma}}\right) \cdot Q,
$$

where, as always, $\sigma=1 /(1-b)$ and $\Lambda=\lambda^{b}$. Inserting (13) and (14) into Eq. (9), we find that

$$
\begin{aligned}
r(h, b)= & E\left[\left(\frac{1}{1+(\tilde{h} \cdot \tilde{\Lambda} \cdot r(\tilde{h}, \tilde{b}))^{\tilde{\sigma}}}\right)^{b}\right]+r(h, b) \cdot h \cdot \Lambda(b) \\
& \cdot E\left[\left(\frac{(\tilde{h} \cdot \tilde{\Lambda} \cdot r(\tilde{h}, \tilde{b}))^{\tilde{\sigma}}}{1+(\tilde{h} \cdot \tilde{\Lambda} \cdot r(\tilde{h}, \tilde{b}))^{\tilde{\sigma}}}\right)^{b}\right]
\end{aligned}
$$

\footnotetext{
${ }^{3}$ The general procedure for solving the Bellman equation is the "method of successive approximations" (see [1]). That will not work for Eqs. (9) and (10) because the set of finite time horizon indirect utility functions generated by the method will not form a monotone sequence the way it does in Bellman's problems.
} 
(where $\tilde{\Lambda}=\Lambda(\tilde{b})$ and $\tilde{\sigma}=\sigma(\tilde{b})$ ), so that $r(\cdot, \cdot)$ must satisfy

$$
r(h, b)=\begin{gathered}
E\left[\left(1 /\left(1+(\tilde{h} \cdot \tilde{\Lambda} \cdot r(\tilde{h}, \tilde{b}))^{\tilde{\sigma}}\right)\right)^{b}\right] \\
\left.\left.\left.1-h \cdot \Lambda(b) \cdot \tilde{\tilde{T}} \cdot \tilde{\bar{\Lambda}} \cdot r((\tilde{h}, \tilde{b}))^{\tilde{\sigma}}\right) /\left(1+(\tilde{h} \cdot \tilde{\Lambda} \cdot r(\tilde{h}, \tilde{b}))^{\tilde{\sigma}}\right)\right)^{b}\right]
\end{gathered} .
$$

Equation (16) shows that if a solution function $R(\cdot)$ of the type shown in line (13) exists, $r(\cdot, \cdot)$ must have the form

$$
r(h, b)=1-h \cdot \bar{\phi}(\bar{\Lambda}(\bar{b}) \cdot \psi(\bar{b}),
$$

where

$$
\begin{aligned}
& \phi(b)=f^{1}(\phi(\cdot), \psi(\cdot), b) \equiv E\left[\left(1+\left(\frac{\tilde{h} \cdot \tilde{\Lambda} \cdot \phi(\tilde{b})}{1-\tilde{h} \cdot \tilde{\Lambda} \cdot \psi(\tilde{b})}\right)^{\tilde{\sigma}}\right)^{-b}\right], \\
& \psi(b)=f^{2}(\phi(\cdot), \psi(\cdot), b) \equiv E\left[\left(\left(\frac{1-\tilde{h} \cdot \tilde{\Lambda} \cdot \psi(\tilde{b})}{\tilde{h} \cdot \tilde{\Lambda} \cdot \phi(\tilde{b})}\right)^{\tilde{\sigma}}+1\right)^{-b}\right]
\end{aligned}
$$

If we can find a solution pair $(\phi(\cdot), \psi(\cdot))$ for the latter two equations, then the functions of lines (13) and (14) will solve Eqs. (9) and (10).

The following proposition supplies sufficiency conditions for the existence of a solution to lines (18) and (19).

Proposition. Let all pairs $(h, b)$ be contained in $H \times B=\left[h_{L}, h_{U}\right] \times$ $\left[b_{L}, b_{U}\right]$. Let $\sigma^{*}=\sigma\left(b_{U}\right), \alpha=h_{L} \cdot \operatorname{Min}_{b \in B}\{\Lambda(b)\}$, and $\theta=h_{U} \cdot \operatorname{Max}_{b \in B}\{\Lambda(b)\}$. Suppose either

(i) $B \subset(0,1)$ and $\theta<1$, or

(ii) $B C(-\infty, 0)$ and $\theta \cdot\left[(1 / \alpha)^{* *}+1\right]^{-b_{L}}=\theta^{*}<1$.

Then Eqs. (18) and (19) have a solution. The solution defines a pair of functions $(R(\cdot), S(\cdot))$ solving Eqs. (9) and (10), and $R(\cdot)$ satisfies Eq. (12).

The Appendix provides a proof.

For those cases in which a solution does exist, the Appendix's fixed-point arguments give few clues about uniqueness. In fact our method of solving Eqs. (9) and (10) does not allow us to rule out solutions with functional forms other than those of lines (13) and (14) anyway. Regardless of whether a solution pair $R(\cdot), S(\cdot))$ is unique, however, we can interpret it as a Nash equilibrium: Suppose $\left(R^{*}(\cdot), S^{*}(\cdot)\right)$ satisfies (9)-(12). If $S^{*}(\cdot)$ will determine the saving of all future cohorts, lines (1) and (3) and the definition of $R(\cdot)$ show that $R(\cdot)$ must be uniquely determined for the present cohort. But, line (12) shows $R(\cdot)=R^{*}(\cdot){ }^{4}$ Thus, $S(\cdot)=S^{*}(\cdot)$ will work on the left-

${ }^{4}$ Note that in terms of the notation we are using here, line (12) requires that $R^{*}(Q, h, b)=$ $\sum_{i=1}^{\infty} h^{i} \cdot E\left[u\left(\widetilde{C}_{i}, b\right)\right]$. 
hand side of line (10). So, if all generations except one adopt the saving function $S^{*}(\cdot)$, the remaining cohort cannot do bettert than to adopt $S^{*}(\cdot)$ for itself. ${ }^{5}$

\section{The Average Propensity to Save}

Section 2 developed a specific class of equilibrium solutions for our economy with intergenerational preference differences. Within the class all optimal aggregate saving functions are linear. We will now compare the implied average propensity to save with that obtained from the conventional analysis outlined in Section 1.

Intergenerational variability of $h$ and $b$ lowers optimal saving for the present generation if for that generation $b>0$, and raises optimal saving if $b<0$. To prove this, for $\phi(\cdot)$ and $\psi(\cdot)$ generated in the proof of our proposition, define

$$
g(z)=\left(\frac{1-z \psi(b)}{\phi(b)}\right)^{\sigma}+z^{\sigma} .
$$

(Note that $\phi(b) \geqslant 0$ and $\psi(b) \geqslant 0$ all $b$ in our solutions.) Then Eq. (14) shows that

$$
S(Q, h, b)=\left[(h \Lambda)^{\sigma} / g(\Lambda h)\right] \cdot Q .
$$

Since Section 1 establishes that the optimal saving rule with a constant discount rate $h$ and elasticity $\sigma$ is $s(Q, h, b)=(h \Lambda)^{\sigma} \cdot Q$, we can compare $S(\cdot)$ and $s(\cdot)$ by comparing $g(h \Lambda)$ and 1 .

Differentiating $g(\cdot)$ with respect to $z$ we find that $g(\cdot)$ is strictly convex if $b>0$ and strictly concave if $b<0$. In each case $g(\cdot)$ has a single critical point, say, $z^{*}$, and

$$
g\left(z^{*}\right)=\left(\phi(b)^{1 / b}+\psi(b)^{1 / b}\right)^{-\sigma b}
$$

If $\tilde{z}$ is a random variable and $G(\cdot)$ is convex, $G(E[\tilde{z}]) \leqslant E[G(\tilde{x})]$. Therefore, if $\tilde{x}(\phi, \psi)=[\tilde{h} \cdot \tilde{\Lambda} \cdot \phi(\tilde{b}) /(1-\tilde{h} \cdot \tilde{\Lambda} \cdot \psi(\tilde{b}))]^{\tilde{s}}$, we have $\left(E\left[(1 /(1+\tilde{x}))^{b}\right]\right)^{1 / b} \leqslant E\left[(1 /(1+\tilde{x}))^{b / b}\right]=E[1 /(1+\tilde{x})]$ and $(E[(\tilde{x} /(1+$ $\left.\left.\tilde{x}))^{b}\right]\right)^{1 / b} \leqslant E[\tilde{x} /(1+\tilde{x})]$. Thus, lines (18) and (19) show that

$$
\phi(b)^{1 / b}+\psi(b)^{1 / b} \leqslant E\left[\frac{1}{1+\tilde{x}(\phi, \psi)}\right]+E\left[\frac{\tilde{x}(\phi, \psi)}{1+\tilde{x}(\phi, \psi)}\right]=1 .
$$

${ }^{5}$ Note that for the solutions derived in the Appendix $\left(R^{*}(\cdot), S^{*}(\cdot)\right)$ will be a perfect Nash equilibrium. In fact, $\left(R^{*}(\cdot), S^{*}(\cdot)\right)$ also constitutes what Blackorby et al. [2], Hammond [5], and Peleg and Yaari [9] call a "sophisticated equilibrium," which is a slightly stronger concept than a Nash equilibrium (see [9]). 
Lines (22) and (23) show that

$$
g\left(z^{*}\right) \geqslant(\leqslant) 1 \quad \text { for } \quad b>(<) 0 .
$$

Convexity and concavity then establish

$$
g(z) \geqslant(\leqslant) 1 \quad \text { for } b>(<) 0 \text { all } z>0 .
$$

So,

$$
S(Q, h, b) \leqslant(\geqslant) s(Q, h, b) \quad \text { if } \quad b>(<) 0 .
$$

The following is an intuitive explanation of the effects of uncertainty on the average propensity to save. The definitions of $v(\cdot)$ (see Section 1) and $V(\cdot)$ (see line (11)) show that $v(Q) \geqslant V(Q)$ all $Q>0: v(Q)$ gives the indirect utility of the present generation under the ideal (for it) conditions that all future cohorts will behave just as it wants them to. ${ }^{6} V(\cdot) \leqslant v(\cdot)$ implies $R(\cdot) \leqslant v(\cdot)$ (see lines (11), (10), and (4)). But $R(\cdot)$ and $v(\cdot)$ have the same functional form with respect to $Q$. Thus, when $b>0$, variability of $h$ and $b$ can lower $R(\cdot)$ only by making $\partial R(Q, h, b) / \partial Q \leqslant \partial v(Q, h, b) / \partial Q$, which, in turn, leads to a lower level of saving. On the other hand, if $b<0, v(\cdot)$ and $R(\cdot)$ are negative so $R(\cdot) \leqslant v(\cdot)$ requires $\partial R(Q, h, b) \partial Q \geqslant \partial v(Q, h, b) / \partial Q$, which means saving will be greater in the variable-parameter case.

\section{CONCLUSION}

Existing papers on the subject of intergenerational preference differences deal for the most part with three issues: identifying various behavioral strategies given changing preferences-see Strotz [14] and Pollak [11]; proving the existence of Nash or "sophisticated" equilibrium solutions for such models-see Phelps and Pollak [10], Peleg and Yaari [9], and Blackorby et al. [2]; and establishing the Pareto efficiency (or, more likely, the lack of Pareto efficiency) of such equilibria or proving the existence of overall orderings which could induce sophisticated behavior through time and which have "sensible" properties - see Phelps and Pollack [10], Blackorby et al. [2], Hammond [5], and Goldman [4]. The present work differs from this literature as follows. Although we study intergenerational preference differences, we assume the differences appear randomly over time. Thus, at any given time, the policymakers in our economy do not have the information-requirement burdens of, for instance, their counterparts in the Peleg-Y aari economy: when calculating equilibrium behavior in the latter situation, policymakers

' We could establish $v(Q) \geqslant V(Q)$ all $Q>0$ rigorously using the inequalities of line (25). 
must know the exact utility functions of all future generations; in our model, policymakers only need to know the present generation's utility function and the distribution function for $(h, b)$ pairs. Our framework also enables us to derive specific comparative static results in Section 3: we show that intergenerational preference variability will cause society's equilibrium average propensity to save to decline (rise) if the present generation's elasticity of marginal utility with respect to consumption $-b-1-$ is between -1 and 0 (is less than -1 ). On the other hand, as is the case in other studies cited above, we do not claim (or expect) that our equilibria are Pareto efficient.

\section{APPENDIX}

The following is a proof of the proposition in Section 2.

Proof. Let $F(\phi(\cdot), \psi(\cdot))=\left(\phi^{*}(\cdot), \psi^{*}(\cdot)\right)$, where $\phi^{*}(b)=f^{1}(\phi(\cdot), \psi(\cdot), b)$ and $\psi^{*}(b)=f^{2}(\phi(\cdot), \psi(\cdot), b)$ (see lines (18) and (19)).

Step 1. Suppose case (i). Let $\Gamma=\{(\phi(\cdot), \psi(\cdot)): \phi(\cdot), \psi(\cdot)$ are continuous; $\phi(b), \psi(b) \in[0,1]$ all $b \in B\}$. Note that $(\phi(\cdot), \psi(\cdot)) \in \Gamma$ implies $h \Lambda(b) \psi(b) \leqslant$ $0<1$ all $(h, b) \in H \times B$.

Define $\phi_{L}(\cdot)=\left[1+(1 /(1-\theta))^{a *}\right]^{-b}$. Let $\gamma=h_{L} \cdot \operatorname{Min}_{b \in B}\{\Lambda(b)\} \cdot \phi_{L}\left(b_{U}\right)$. Define $\psi_{L}(\cdot)=\left[(1 / \gamma)^{\sigma *}+1\right]^{-b}$. Then $\phi_{L}(b), \psi_{L}(b)>0$ all $b \in B$. Define $\Gamma^{*}=\left\{(\phi(\cdot), \psi(\cdot)) \in \Gamma: \phi(b) \geqslant \phi_{L}(b), \psi(b) \geqslant \psi_{L}(b)\right.$ all $\left.b \in B\right\}$. Then lines (18)-(19) show $F\left(\Gamma^{*}\right) \subset \Gamma^{*}$.

Step 2. Suppose case (ii). Define $\Gamma=\{(\phi(\cdot), \psi(\cdot)): \phi(\cdot), \psi(\cdot)$ are continuous; $h \Lambda(b) \psi(b) \leqslant \theta^{*}$ all $(h, b) \in H \times B$; and, $\phi(b), \psi(b) \geqslant 1$ all $\left.b \in B\right\}$. Let $(\phi(\cdot), \psi(\cdot)) \in \Gamma$ and $\left(\phi^{*}(\cdot), \psi^{*}(\cdot)\right)=F(\phi(\cdot), \psi(\cdot))$. Then $\phi^{*}(b), \psi^{*}(b) \geqslant 1$ all $b \in B$ (see lines (18)-(19)).

Let $\psi_{U}(b)=\left[(1 / \alpha)^{o *}+1\right]^{-b}$. Then $\psi(b) \leqslant \psi_{U}(b)$ all $b \in B$ implies $h \Lambda(b) \psi(b) \leqslant \theta^{*}<1$ all $(h, b) \in H \times B$ by hypothesis (ii).

Define $\gamma^{*}=\left[1 /\left(1-\theta^{*}\right)\right]^{\sigma *}$ and $\beta=-b_{L} /\left(1-b_{L}\right)$. Then $\beta \in(0,1)$. Define $g(x)=1+\gamma^{*} x^{\beta}$. Then $\lim _{x \rightarrow \infty} g(x) / x=0$. So, there exists $x^{*} \geqslant 1$ with $g\left(x^{*}\right)<x^{*}$. Fix such an $x^{*}$.

Define $\phi_{U}(b)=x^{*^{-b}}$. Define $\Gamma^{*}=\left\{(\phi(\cdot), \psi(\cdot)) \in \Gamma: \phi(b) \leqslant \phi_{U}(b), \psi(b) \leqslant\right.$ $\psi_{U}(b)$ all $\left.b \in B\right\}$. Let $(\phi(\cdot), \psi(\cdot)) \in \Gamma^{*}$ and $\left(\phi^{*}(\cdot), \psi^{*}(\cdot)\right)=F(\phi(\cdot), \psi(\cdot))$. Then line (19) shows $\psi^{*}(b) \leqslant \psi_{U}(b)$ all $b \in B$. Line (18) shows $\phi^{*}(b) \leqslant E[\{1+$ $\left.\left.\gamma^{*}\left(\phi_{U}(\tilde{b})\right)^{\delta}\right\}^{-b}\right] \leqslant\left\{1+\gamma^{*} x^{*^{*}}\right\}^{-b}=\left\{g\left(x^{*}\right)\right\}^{-b}<x^{*^{-b}}=\phi_{U}(b)$. So, $F\left(\Gamma^{*}\right) \subset \Gamma^{*}$.

Step 3. In case (i) let $\Gamma^{*}$ be defined as in Step 1 ; in case (ii) let $\Gamma^{*}$ be defined as in Step 2. Let $(\phi(\cdot), \psi(\cdot)) \in \Gamma^{*}$. Then $\phi(b), \psi(b)>0$ all $b \in B$ and $h \Lambda(b) \psi(b)<1$ all $(h, b) \in H \times B$. So, if we adopt the uniform norm on $\Gamma^{*}-(\phi(\cdot), \psi(\cdot)), \quad(\bar{\phi}(\cdot), \bar{\psi}(\cdot)) \in \Gamma^{*} \quad$ implies $\quad|(\phi(\cdot), \psi(\cdot))-(\bar{\phi}(\cdot), \bar{\psi}(\cdot))|=$ 
$\operatorname{Max}\left\{\sup _{b \in B}|\phi(b)-\bar{\phi}(b)|, \quad \sup _{b \in B}|\psi(b)-\bar{\psi}(b)|\right\}$-then $F(\cdot)$ is continuous on $\Gamma^{*} . \Gamma^{*}$ is convex. Thus, Schauder's fixed-point theorem (see Smart [13]) shows that $F(\cdot)$ will have a fixed point on $\Gamma^{*}$ if $F\left(\Gamma^{*}\right)$ is contained in a compact subset of $\Gamma^{*}$. Each fixed point for $F(\cdot)$ defines a solutions to Eqs. (18) and (19).

Step 4. Since $(\phi(\cdot), \psi(\cdot)) \in \Gamma^{*}$ implies $\phi(b)$ and $1-h \Lambda(b) \psi(b)$ are bounded away from 0 for all $(h, b) \in H \times B$, for each case (i.e., case (i) and case (ii)) there exists a finite constant $\delta$ such that $\left|\partial f^{1}(\phi(\cdot), \psi(\cdot), b) / \partial b\right|$, $\left|\partial f^{2}(\phi(\cdot), \psi(\cdot), b)\right| \partial b \mid<\delta$ all $(\phi(\cdot), \psi(\cdot)) \in \Gamma^{*}$ and $b \in B$. Then $\left|b-b^{*}\right|<$ $\epsilon / \delta$ implies $\mid\left(\phi^{*}(b)-\phi^{*}\left(b^{*}\right)|,| \psi^{*}(b)-\psi^{*}\left(b^{*}\right) \mid<\epsilon\right.$ if $(\phi(\cdot), \psi(\cdot)) \in \Gamma^{*}$ and $\left(\phi^{*}(\cdot), \psi^{*}(\cdot)\right)=F(\phi(\cdot), \psi(\cdot))$. So, $\Gamma^{* *}=F\left(\Gamma^{*}\right)$ is equicontinuous. Thus, $\Gamma^{* * *}=$ Closure ( $\Gamma^{* *}$ ) is also. So, Ascoli's theorem (see Munkres [8]) shows $\Gamma^{* * *}$ is compact. Thus, Step 3 shows $F(\cdot)$ has a fixed point on $\Gamma^{*}$.

Step 5. Let $(\phi(\cdot), \psi(\cdot)) \in \Gamma^{*}$ be a fixed point for $F(\cdot)$. Use lines (13), (14), and (17) to define a pair $(R(\cdot), S(\cdot))$ from $(\phi(\cdot), \psi(\cdot))$. Then $(R(\cdot), S(\cdot))$ solves Eqs. (9) and (10). In terms of the notation of line (12), Eq. (9) shows $R(Q, h, b)=\sum_{i=1}^{T} h^{i} \cdot E\left[u\left(\tilde{C}_{i}, b\right)\right]+h^{T+1} \cdot E\left[R\left(\tilde{Q}_{T+1}, \tilde{h}_{T+1}, \tilde{b}_{T+1}\right)\right]$ any $T>1$. Thus, if we can show $h^{T+1} \cdot E\left[R\left(\tilde{Q}_{T+1}, \tilde{h}_{T+1}, \tilde{b}_{T+1}\right)\right] \rightarrow 0$ for each $Q>0$ as $T \rightarrow \infty$, we will have established that $R(\cdot)$ satisfies line (12).

Suppose case (i). Then for all $(h, b) \in H \times B, r(h, b) \geqslant 0$ (see line (17)) because $\phi(b) \geqslant 0$ and $1>\theta \geqslant \theta \cdot \psi(b)$. Let $r_{U}=1 /(1-\theta)$. Then $0 \leqslant r(h, b) \leqslant r_{U}$ all $(h, b) \in H \times B$. Hence, $0 \leqslant h^{T+1} \cdot E\left[R\left(\tilde{Q}_{T+1}, \tilde{h}_{T+1}\right.\right.$, $\left.\left.\tilde{b}_{T+1}\right)\right] \leqslant h^{T+1} \cdot b \cdot r_{U} \cdot Q^{b} \cdot E\left[\left(\tilde{s}_{0} \cdot \tilde{s}_{1} \cdot \cdots \cdot \tilde{s}_{T}\right)^{b}\right] \cdot \Lambda(b)^{T+1}$, where $\quad \tilde{s}_{i}=$ $\left(\tilde{h}_{i} \cdot \Lambda\left(\tilde{b}_{i}\right) \cdot r\left(\tilde{h}_{i}, \tilde{b}_{i}\right)\right)^{\tilde{\sigma}_{i}}\left[\left[1+\left(\tilde{h}_{i} \cdot \Lambda\left(\tilde{b}_{i}\right) \cdot r\left(\tilde{h}_{i}, \tilde{b}_{i}\right)\right)^{\tilde{\sigma}_{i}}\right], \quad \tilde{\sigma}_{i}=1 /\left(1-\tilde{b}_{i}\right) \quad\right.$ all $i=0, \ldots, T$. Then $\tilde{s}_{i} \in[0,1)$, so $0 \leqslant h^{T+1} \cdot E\left[R\left(\widetilde{Q}_{T+1}, \tilde{h}_{T+1}, \tilde{b}_{T+1}\right)\right] \leqslant \theta^{T+1}$. $r_{U} \cdot Q^{b}$. But, $\theta^{T+1} \cdot r_{U} \cdot Q^{b} \rightarrow 0$ as $T \rightarrow \infty$ for any $Q \geqslant 0$.

Suppose case (ii). Then $r(h, b) \geqslant 0$ (see line (17)) for all $(h, b) \in H \times B$. Let $r_{U}=\operatorname{Max}_{b \in B} \phi_{U}(b) /\left(1-\theta^{*}\right)$. Then $r(h, b) \leqslant r_{U}$ all $(h, b) \in H \times B$. Using the definition of $\tilde{s}_{i}$ from the preceding paragraph, $0 \geqslant h^{T+1} \cdot E\left[R\left(\tilde{Q}_{T+1}\right.\right.$, $\left.\left.\tilde{h}_{T+1}, \tilde{b}_{T+1}\right)\right] \geqslant h^{T+1} \cdot b \cdot r_{U} \cdot Q^{b} \cdot E\left[\left(\tilde{s}_{0} \cdot \tilde{s}_{1} \cdot \cdots \cdot \tilde{s}_{T}\right)^{b}\right] \cdot A(b)^{T+1} \geqslant \theta^{T+1} \cdot b \cdot$ $r_{U} \cdot Q^{b}$. But, $\left|\theta^{T+1} \cdot b \cdot r_{U} \cdot Q^{b}\right| \rightarrow 0$ as $T \rightarrow \infty$ for each $Q>0$. Q.E.D.

\section{REFERENCES}

1. R. E. Bellman, "Dynamic Programming," Princeton Univ. Press, Princeton, N. J., 1957.

2. C. Blackorby, D, Nissen, D. Primont, and R. R. Russell, Consistent intertemporal decision making, Rev. Econ. Stud. 40 (1973), 239-248.

3. D. CAss, Optimal growth in an aggregative model of capital accumulation: A turnpike theorem, Econometrica 34 (1966), 833-850.

4. S. M. Godlman, Intertemporally inconsistent preferences and the rate of consumption, Ecunometrica 47 (1979), 621-626. 
5. P. J. Hammond, Changing tastes and coherent dynamic choice, Rev. Econ. Stud. 43 (1976), 159-174.

6. T. Koopmans, Stationary ordinal utility and impatience, Econometrica 28 (1960), 282-308.

7. D. Levhari and T. N. SRinivasan, Optimal savings under uncertainty, Rev. Econ. Stud. 36 (1969), 153-164.

8. J. R. Munkres, "Topology: A First Course," Prentice-Hall, Englewood Cliffs, N.J., 1975.

9. B. Peleg and M. E. YaAri, On the existence of a consistent course of action when tastes are changing, Rev. Econ. Stud. 40 (1973), 391-402.

10. E. S. Phelps AND R. A. Pollak, On second-best national saving and game-equilibrium growth, Rev. Econ. Stud. 35 (1968), 185-199.

11. R. A. Pollak, Consistent planning, Rev. Econ. Stud. 35 (1968), 201-208.

12. F. P. RAMSEY, A mathematical theory of saving, Econ. J. 38 (1928), 543-559.

13. D. R. Smart, "Fixed Point Theorems," Cambridge Univ. Press, London, 1974.

14. R. H. Strotz, Myopia and inconsistency in dynamic utility maximization, Rev. Econ. Stud. 23 (1956), 165-180. 